Paper

\title{
The Mechanical and Thermal Properties of Porous Zirconia Ceramics Fabricated through a Solid-state Foaming Method
}

\author{
Masaaki Hanao, Hidetaka Hayashi and Akira Kishimoto \\ Division of Chemistry and Biochemistry, Graduate School of Natural Science and Technology, Okayama University, \\ 3-1-1 Tsushima-naka, Okayama 700-8530, Japan.
}

Received May 7, 2008

\begin{abstract}
SYNOPSIS
We have already fabricated ceramic mono-foams by expanding once-sintered dense shells utilizing the superplastic deformation of $3 \mathrm{YSZ}, 8 \mathrm{YSZ}$, alumina and titania based ceramics. In the present study, granular coating method was applied to make a porous ceramics through the superplastically foaming method based on 3YSZ. Both mechanical strength and thermal conductivity were measured and compared with those of fully densified as well as conventional porous $3 \mathrm{YSZ}$ ceramics. Porous ceramics with porosity over $35 \%$ can be fabricated with less than 15 vol\% of foam agent. The mechanical strength and thermal conductivity were superior or comparable to those for conventionally fabricated porous ceramics with the same porosity level.
\end{abstract}

KEY WORDS

zirconia, porous ceramics, foam, superplasticity

\section{Introduction}

Porous ceramics are widely used as filters, catalyst supports, lightweight structural materials, fireproof materials, sound insulators, etc.; these applications take advantage of general ceramic properties such as nonflammability, chemical stability, and high mechanical strength ${ }^{1)}$. For use as a filter and a catalytic support, porous ceramics contain open pores that are selectively introduced by an organic binder method $^{2)}$ or a pore forming additive method ${ }^{33}$; these open pores result in insufficient sintering. On the other hand, porous ceramics with predominantly closed pores are preferable for use in lightweight structural and fireproof material applications and as sound insulators.

One of the authors has developed a novel pore-incorporation method utilizing the superplasticity of ceramics ${ }^{4-10)}$. With this method, a foam agent is introduced into a matrix powder, and at approximately the sintering temperature, the evolving gas created from the foam agent produces a pressure that expands the pore walls. After pore expansion, the ceramic is fully densified to produce a dense pore wall. Then, closed pores are selectively introduced. The resulting foam has high strength, a high level of gas tightness, and provides good thermal insulation.

We have already demonstrated fabricating ceramic foams based on $3 \mathrm{~mol} \%$ yttria stabilized zirconia (3YSZ $)^{4,5)}, 8 \mathrm{~mol} \%$ yttria stabilized zirconia $(8 Y S Z)^{6)}$, alumina ${ }^{7,8)}$, and titania ${ }^{9)}$.
These foams mainly have a mono-foam structure that contains a macroscopic closed pore in a pellet. Since the fabrication process of the mono-foam includes powder compaction of the foam agent, this type of foam production is not suitable for porous ceramics containing a significant amount of micro-pores, which are advantageous for applications requiring good thermal insulation and high mechanical strength properties.

Employing a screen printing method, we have fabricated a ceramic foam containing several or tens of pores in a pellet ${ }^{10)}$. With this method we can control the size and position of a pore by adjusting the amount and location of the foam agents. However, the productivity scale of this method cannot fabricate enough foam for large structural material applications, such as thermal insulator walls. In the present study we employ a granular coating method in which the cyclic patterning of a foam agent can be easily fabricated in matrix powders.

We employed 3YSZ, mainly because its superplastic deformation allows ceramic foam fabrication without additives. Zirconia ceramics are also known to be refractory materials with high mechanical strength and low thermal conductivity. In the present study, a granular coating method is used to make porous ceramics based on the superplasticity foaming of 3YSZ. Preliminary measurements on both the mechanical strength and thermal conductivity are obtained 
and compared with those of fully densified and conventional porous 3 YSZ ceramics.

\section{Experimental Procedure}

To arrange the foam agents periodically, granular foam agents were coated by a matrix powder to produce core/ shell-structured granules. Fig. 1 shows a schematic illustration of the fabrication of the core/shell-structured granules. First, spherical core granules composed of foam agent powders were fabricated by ball milling fine $\beta$-SiC powders (Grade-UF, Ibiden, Aichi, Japan). Uniformly sized $(\sim 250 \mu \mathrm{m})$ core granules were selected by passing them through stainless steel meshes.

Next, a 1 wt\% methylcellulose aqueous solution was sprayed on the core granules to facilitate the adhesion of the matrix powder. The surface-treated core granules were placed into a polyethylene pot (inner diameter $80 \mathrm{~mm}$ ) with the matrix powders (TZ3, Tosho, Tokyo, Japan) and polyethylene balls. The matrix powders had been treated by ultrasonic irradiation to eliminate agglomeration. Through dry coating in a ball mill, core/shell-structured granules with various matrix powder coating layers were fabricated (Fig. 2). These two-layered granules were put into a $\phi 20$ steel die, and then were subjected to a uniaxial compression of $50 \mathrm{MPa}$. After cold isostatic pressing at $200 \mathrm{Mpa}$, powder compacts were heated in air at a rate of $800^{\circ} \mathrm{C} / \mathrm{h}$ to $1600^{\circ} \mathrm{C}$ and maintained at that temperature for $8 \mathrm{~h}$ to densify the matrix. Next, the pores were expanded by the gas that evolved from the foam agent.

The apparent density was measured by the Archimedes method using water as the medium. The mechanical strength was measured by a three-point flexure machine (EZ-test, Shimadu, Kyoto, Japan), where a rectangular bar $(2.6 \times 2.6 \times 10 \mathrm{~mm})$ with a span length of $9 \mathrm{~mm}$ was used. Thermal conductivity $(\lambda)$ was measured on a disk sample by the laser flush method (Netch, Germany). Pellet samples were coated with a thin graphite layer to improve laser absorption. To calculate the thermal diffusivity $(\alpha)$, we analyzed the back face temperature-time behavior. The thermal conductivity was then obtained with the expression

$$
\lambda=\alpha \rho c
$$

where $\rho$ is the density and $c$ is the specific heat of the material. The specific heat was obtained from the literature.

\section{Results and Discussion}

Fig. 3 (a) shows the granulated $\beta$-SiC, the foam agent. Nearly spherical granules of uniform size were fabricated. Various amounts of 3YSZ matrix powders were adhered to the core $\mathrm{SiC}$ granules to make $\mathrm{SiC}$ (core)/3YSZ(matrix) composite granules and are shown in Figs. 3 (b)-(d). Compactions of the composite granules used in the present study were all well enough to lead periodically arranged powder compacts. Smaller size core granule is thought to be preferable taking into account of the mechanical and thermal properties, however, cores smaller than $250 \mu \mathrm{m}$ did not give a stable composite granule.

Fig. 4 shows optical micrographs of (a) cross-sectional and (b) surface views of the sintered body, and SEM photos of (c) 3YSZ-based porous ceramics fabricated by superplastically foamed ceramics and (d) a conventional ceramic that was insufficiently sintered (d), respectively. As shown in the surface micrographs, no open pores were observed, suggesting that closed pores were selectively introduced.

Fig. 5 shows the relationship between porosity and the amount of foam agent. Results for the relationship between

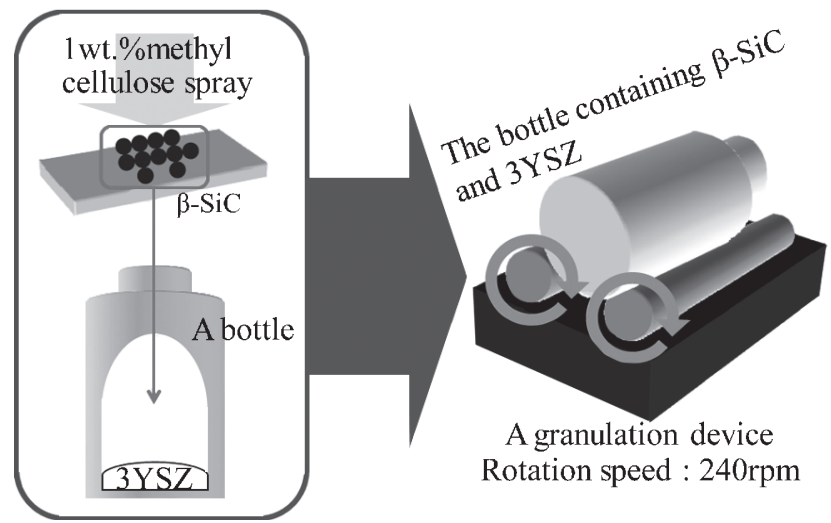

Fig.2 Schematic illustration of granulation of $\beta$-SiC coated with 3 YSZ.

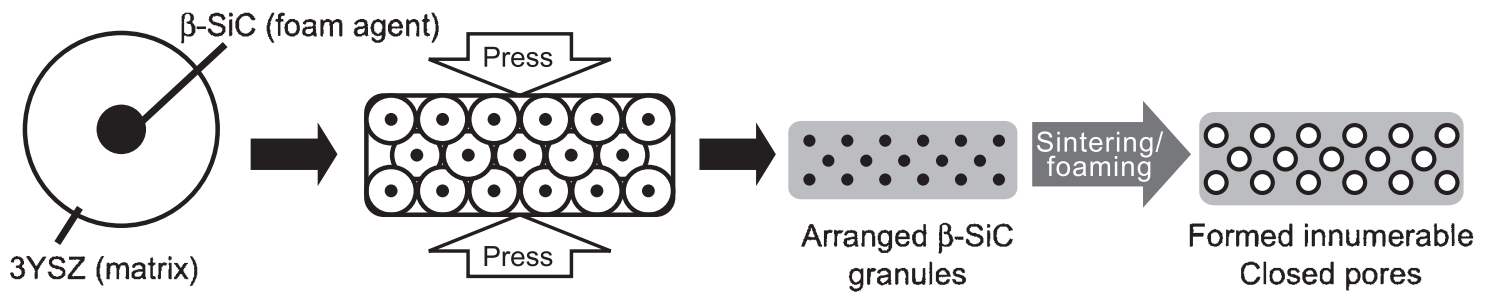

Fig.1 Schematic illustration of fabrication of porous 3 YSZ ceramics using superplastic deformation 

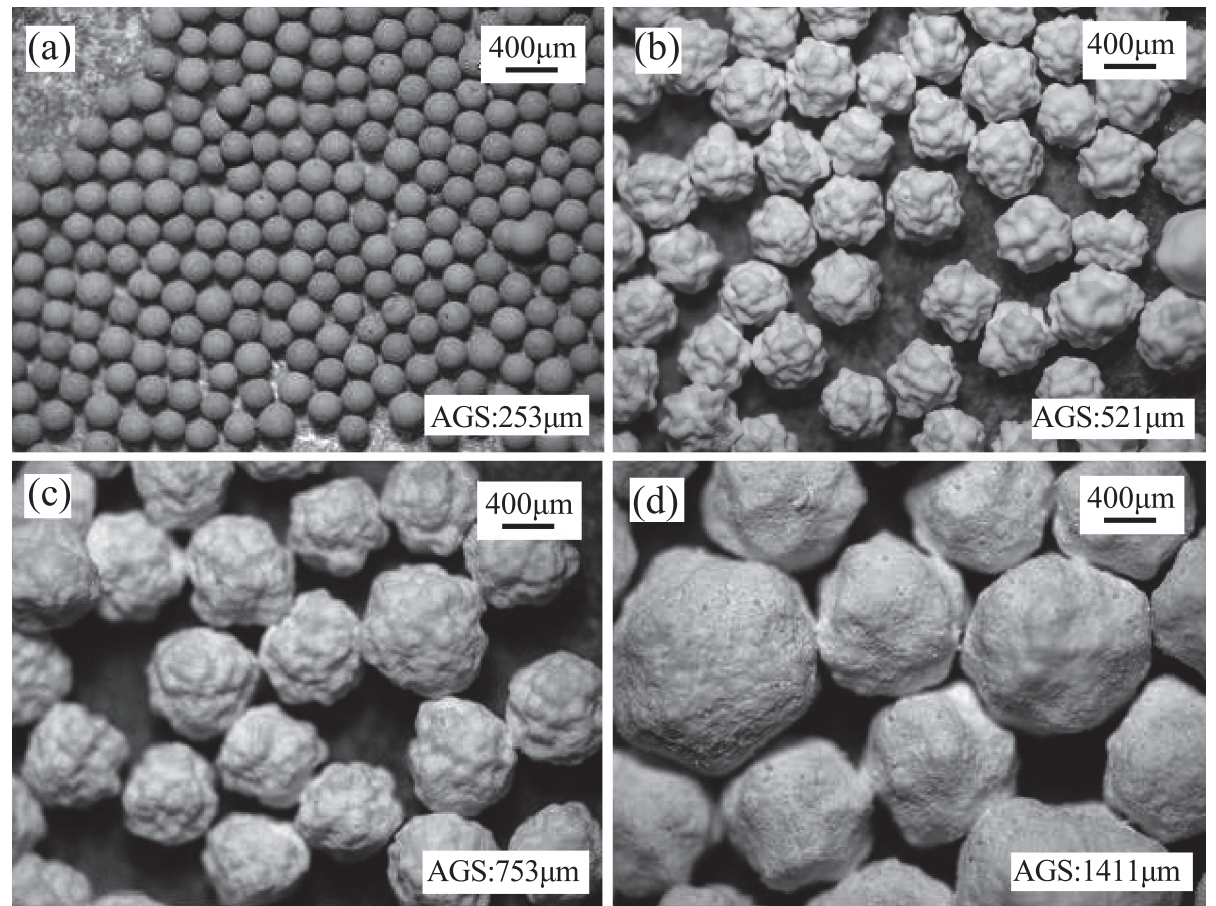

Fig.3 Photograph of particles (a) $\beta$-SiC granule (b)-(d) $\beta$-SiC granules coated with various amount of 3 YSZ (AGS = average grain size)
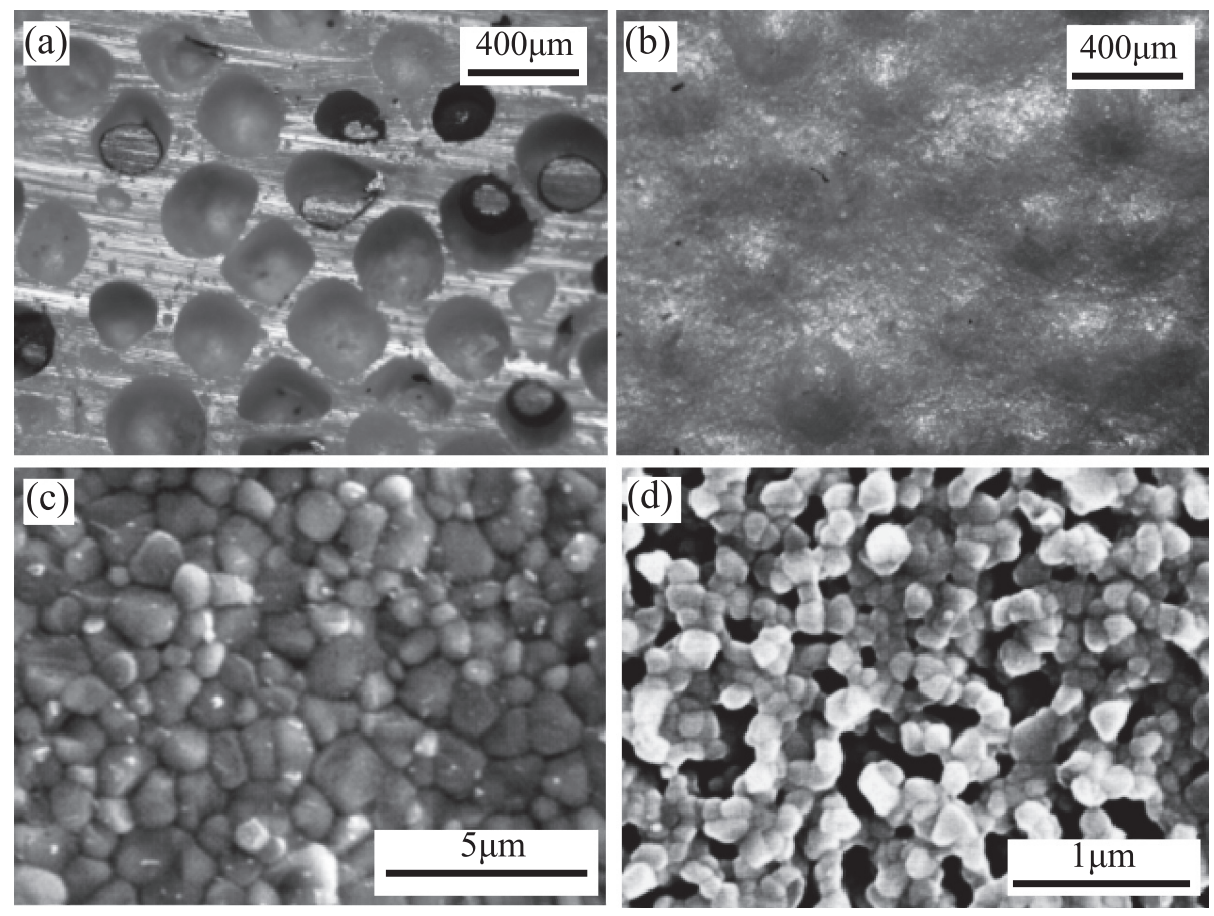

Fig.4 Optical micrograph of cross section (a) and surface (b), (c) of superplastisity foamed 3YSZ ceramics. (d): surface photo of partically sintered 3 YSZ ceramics.

the porosity and a typical pore former, polymethyl methacrylate (PMMA), which decomposes at approximately $400^{\circ} \mathrm{C}$, are also shown in the same figure ${ }^{11)}$. Compared with PMMA, a relatively small amount of foam agent $(\mathrm{SiC})$ gave rise to greater porosity.
This result suggests that the gas evolution from the foam agent occurs after the densification of the pore wall and leads to pore expansion, thus utilizing the superplastic deformation of the matrix ${ }^{4,5)}$. In other words, the foam agent must be shielded from the ambient air to lead the gas 


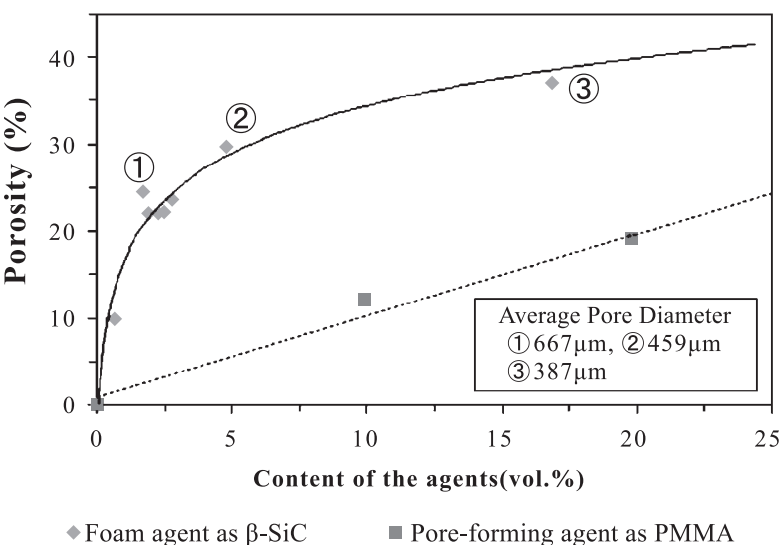

Fig.5 Dependence of porosity on the content of foam agent (SiC, vol\%) in superplastisity foamed 3YSZ ceramics (Relationship between pore former (PMMA) and porosity is also shown as a reference).

evolvable active oxidation ${ }^{4)}$ otherwise a stable passive oxidation to form solid silica occurs in atmospheric air at the present heating temperature. On the other hand, PMMA decomposes far below the sintering temperature, and the vacant space left by the pore former should shrink when sintering is applied. The resultant pore sizes from the uniformly sized pore formers are nearly constant and give a liner relationship between the amount of pore former and total porosity, as shown in Fig. 5.

In the case of $\beta-\mathrm{SiC}$, the porosity increases parabolically and saturates with a ratio of foam agent $(\mathrm{SiC})$ to matrix. The porosity should be linearly related to the population of pores assuming that the pore size remains constant. This parabolic relation suggests that the pore size became small with an increase in the amount of foam agent because a pore formed in a pellet should correspond one-to-one with a granulated foam agent. In the present study, the driving force to expand a pore is thought to be constant, irrespective of the composition of the foam agent, because the amount of evolved gas from the same-sized granulated foam agent should be identical.

When the amount of foam agent exceeds a constant value, pore growth can be suppressed by certain factors. One of the probable factors is the interference between the neighboring pores because the radial expanding forces counteract each other.

Fig. 6 shows the three-point bending strength of superplastically foamed porous ceramics. The strength of fully densified ceramics from the same starting matrix powder is shown in the same figure. In this study, an ultrasonic treatment was conducted on the starting matrix powder to obtain fine powders. This method is useful for

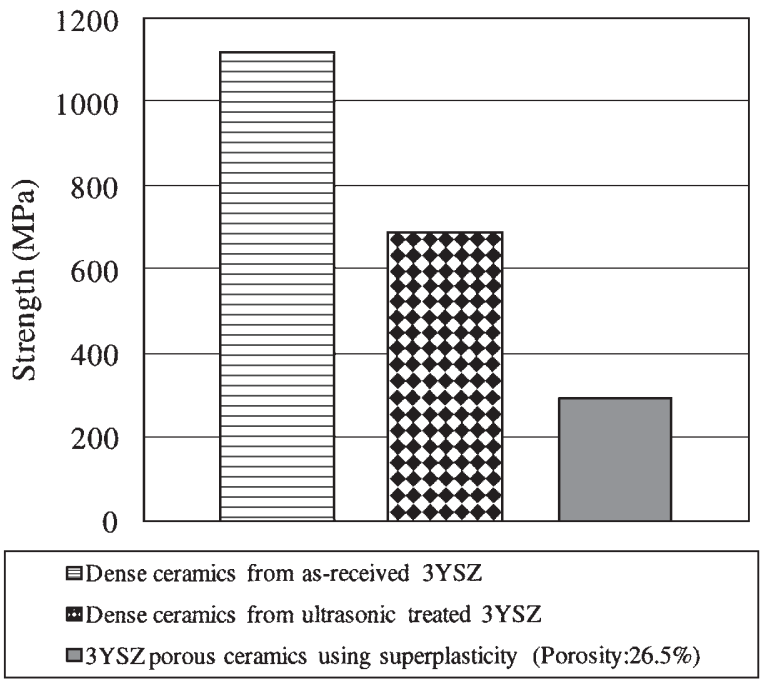

Fig.6 Bending strength of superplastisity foamed 3 YSZ ceramics

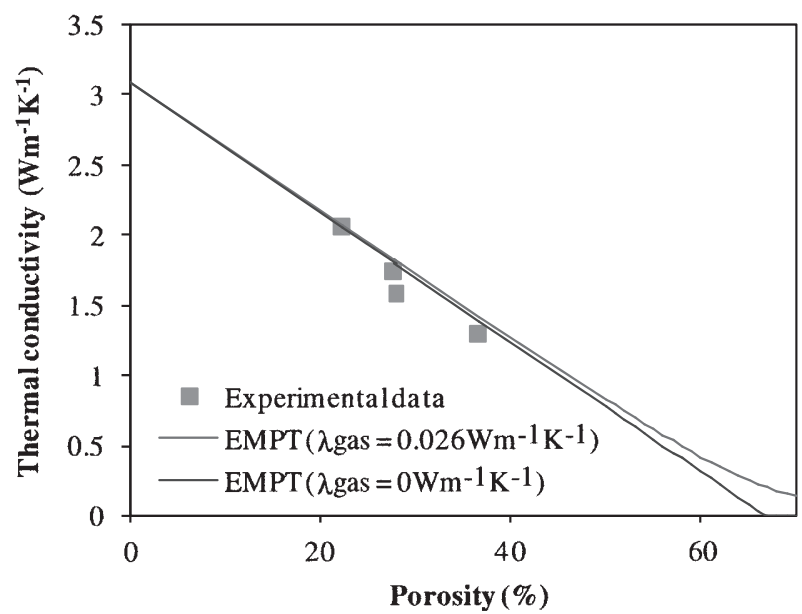

Fig.7 Dependence of thermal conductivity on the porosity of superplastisity foamed 3 YSZ ceramics.

granulation coating. After sintering, the ceramic with ultrasonically treated powders had a lower bending strength than that of the ceramic with as-received powders. The superplastically foamed porous ceramics were made from the ultrasonically treated powders. Fully densified 3YSZ formed from a similarly treated powder was used as a reference. The mechanical strength of superplastically foamed porous ceramics with a porosity of $26.5 \%$ was 289 MPa on average. This strength value was $42 \%$ of that for fully densified ceramics (688 MPa). Conventional porous ceramics with the same $26.5 \%$ porosity level had been reported with strengths less than $30 \%$ of those for fully densified ceramics ${ }^{11}$, which accord with the traditional model or exponential relationship between porosity and mechanical strength ${ }^{12)}$. The relatively large mechanical 
strength for the superplastically foamed porous ceramic in this study is probably ascribed to strong intergrain bonding based on the fully densified 3 YSZ followed by the pore expansion.

Fig. 7 shows the thermal conductivity of porous ceramics fabricated by superplasticity foaming using the $3 \mathrm{YSZ}$ coated granules on a $\beta$-SiC core with an average diameter of $253 \mu \mathrm{m}$. The porous solid is considered to be a twophase system composed of a dense solid and air. Several expressions have been proposed to calculate the thermal conductivity of porous materials; all are based on the geometrical approximation of the microstructure -the spatial distribution of pores in the dense matrix ${ }^{13-20)}$. Selection of the appropriate expression is necessary to describe the profile of experimental results.

In the present study, the effective medium percolation theory (EMPT) was chosen to describe the results. In this theory, a random mixture of spherical particles of two different phases is considered. The neighborhood of each particle is assumed to exhibit the thermal conductivity, which characterizes the mixture ${ }^{17,20)}$,

$$
\begin{aligned}
\lambda_{\text {eff }}=1 / 4 & {\left[\lambda_{\mathrm{p}}\left(3 v_{\mathrm{p}}-1\right)+\lambda_{\mathrm{s}}\left(3 v_{\mathrm{s}}-1\right)\right.} \\
+ & \left.\left(\left[\lambda_{\mathrm{p}}\left(3 v_{\mathrm{p}}-1\right)+\lambda_{\mathrm{s}}\left(3 v_{\mathrm{s}}-1\right)\right]^{2}+8 \lambda_{\mathrm{p}} \lambda_{\mathrm{s}}\right)^{1 / 2}\right]
\end{aligned}
$$

where $\lambda_{\text {eff }}$ is the effective thermal conductivity; $\lambda_{\mathrm{s}}$ and $\lambda_{\mathrm{p}}$ are the thermal conductivities of the solid matrix and the pores, respectively; and $v_{\mathrm{s}}$ and $v_{\mathrm{p}}$ are the corresponding volume fractions of these phases.

Literature values of the thermal conductivity in air and a vacuum $\left(\lambda_{\mathrm{p}}=0.026 \mathrm{Wm}^{-1} \mathrm{~K}^{-1}, 0 \mathrm{Wm}^{-1} \mathrm{~K}^{-1}\right)$ and experimental results for fully dense $3 \mathrm{YSZ}\left(\lambda_{\mathrm{s}}=3.1 \mathrm{Wm}^{-1} \mathrm{~K}^{-1}\right)$ were used to calculate a theoretical profile, illustrated in Fig. 7. The experimental results were in accord with the theoretical profile and suggest that the present microstructure follows the EMPT.

In summary, we analyzed the composition of the gas phase in the closed pore of a superplastically foamed single foam. The analysis revealed the solidification of an evolved gas at room temperature ${ }^{4)}$. Accompanied by the solidification of the gas phase, the inner pressure of the closed pore should be lower and should lead to a smaller thermal conductivity value.

According to the theoretical line shown in Fig. 7, the thermal conductivities of ceramics with vacuum pores and ceramics with air pores were in good agreement up to $40 \%$ porosity. A discrepancy gradually appeared when the porosity rose to approximately $50 \%$, and the discrepancy became much greater when the porosity was over $60 \%$.
We could not fabricate a porosity larger than $38 \%$. A further reduction in the thermal conductivity is expected when the porosity exceeds $60 \%$ based on the pores of reduced pressure.

\section{Conclusions}

Ggranular coating method was applied to make a porous ceramics through the superplastically foaming method based on 3YSZ. Both mechanical strength and thermal conductivity were measured and compared with those of fully densified as well as conventional porous 3 YSZ ceramics. Porous ceramics with porosity over $35 \%$ can be fabricated with less than 15 vol\% of foam agent. The mechanical strength and thermal conductivity were superior or comparable to those for conventionally fabricated porous ceramics with the same porosity level.

\section{References}

1) S.L. Suib: "Sorption, catasysis, and separation by design", Chemical Innovation, 30(2000)27-33.

2) Z.Y. Deng, J.F. Yang, Y. Beppu, M. Ando, and T. Ohji: "Effect of Agglomeration on Mechanical Properties of Porous Zirconia Fabricated by Partial Sintering", J. Am. Ceram. Soc., 85(2002)1961-1965.

3) R. Barea, M.I. Osendi, P. Miranzo, and J.M.F. Ferreira: "Fabrication of Highly Porous Mullite Materials", J. Am. Ceram. Soc., 88(2005)777-779.

4) A. Kishimoto, T. Higashiwada, H. Asaoka, and H. Hayash: "The exploitation of superplasticity in the successful foaming of ceramics following sintering", Adv. Eng. Mater., 8(2006)708-711.

5) T. Higashiwada, H. Asaoka, H. Hayashi, and A. Kishimoto: "Effect of additives on the pore evolution of zirconia based ceramic foams after sintering", J. Eur. Ceram. Soc., 27(2007)2217-2222.

6) Y. Hashida, H. Hayashi, and A. Kishimoto: "Fabrication of Solid State Foams Based on Full Stabilized Zirconia Facilitating the Superplasticity with Dispersoids", J. Jpn. Soc. Powder Powder Metallurgy, 54(2007)740-743.

7) A. Kishimoto, M. Obata, H. Asaoka, and H. Hayashi: "Fabrication of alumina-based ceramic foams utilizing superplasticity", J. Eur. Ceram. Soc., 27(2007)41-45.

8) M. Obata, H. Hayashi, and A. Kishimoto: "Alumina based mono-foams utilizing the superplastic deformation facilitated by addition of magnesia or magnesium aluminate spinel", J. Alloys Compd., in press.

9) A. Kishimoto, M. Obata, K. Waku, and H. Hayashi: "Mechanical and electrical properties of superplastically 
foamed titania based ceramics", Ceram. Intern., in press

10) A. Kishimoto, T. Higashiwada, M. Takahara, and H. Hayashi: "Solid state foaming and free-forming of closed pore utilizing the superplasticity of zirconia ceramics", Mater. Sci. Forum, 544-545(2007)641-644.

11) A.K. Gain, H.Y. Song, and B.T. Lee: "Microstructure and mechanical properties of porous yttria stabilized zirconia ceramic using poly methyl methacrylate powder", Scr. Mater., 54(2006)2081-2085.

12) R.L. Coble and W.D. Kingery: "Effect of Porosity on Physical Properties of Sintered Alumina", J. Am. Ceram. Soc., 39(1956)377-385.

13) L. Rayleigh: "On the influence of obstacles arranged in rectangular order upon the properties of medium", Phil. Mag., 34(1892)481-507.

14) H.W. Russel: "Principle of heat flow in porous insulators", J. Am. Ceram. Soc., 18(1935)1-5.

15) Z. Hashin and S. Shtrikman: "A variational approach to the theory of the effective magnetic permeability of multiphase materials", J. Appl. Phys., 33(1962)31253131 .

16) R. Landauer: "The electrical resistance of binary metallic mixtures", J. Appl. Phys., 21(1952)779-784.

17) D.G. Ast: "Evidence for percolation-controlled conductivity in amorphous $\mathrm{As}_{\mathrm{x}} \mathrm{Te}_{1-\mathrm{x}}$ films", Phy. Rev. Lett., 33(1974)1042-1045.

18) B. Schulz: "Thermal conductivity of porous and highly porous materials", High Temp.-High Press., 13(1981) 649-660.

19) A. Bjorneklett, L. Haukeland, J. Wigren, and H. Kristiansen: "Effective medium theory and the thermal conductivity of plasma-sprayed ceramic coatings", J. Mater. Sci., 29(1994)4043-4050.

20) B. Nait-Ali, K. Haberko, H. Vesteghem, J. Absi, and D.S. Smith: "Thermal conductivity of highly porous zirconia", J. Eur. Ceram. Soc., 26(2006)3567-357. 